\title{
Transient acute kidney injury after major abdominal surgery increases chronic kidney disease risk and 1- year mortality
}

\section{AUTHOR(S):}

Mizota, Toshiyuki; Dong, Li; Takeda, Chikashi;

Shiraki, Atsuko; Matsukawa, Shino; Shimizu, Satoshi; Kai, Shinichi

\section{CITATION:}

Mizota, Toshiyuki ...[et al]. Transient acute kidney injury after major abdominal surgery increases chronic kidney disease risk and 1-year mortality. Journal of Critical Care 2019, 50: $17-22$

\section{ISSUE DATE:}

2019-04

\section{URL:}

http://hdl.handle.net/2433/243863

\section{RIGHT:}

(c) 2018. This manuscript version is made available under the CC-BY-NC-ND 4 . license

http://creativecommons.org/licenses/by-nc-nd/4.0/; The full-text file will be made open to the public on 1 April 2020 in accordance with publisher's 'Terms and Conditions for Self-Archiving'.; This is not the published version. Please cite only the published version.; この論文は出版社版でありません。引用の際には出版社版をご確認ご利用ください。 
Transient acute kidney injury after major abdominal surgery increases chronic kidney disease risk and 1-year mortality

Toshiyuki Mizota*, Li Dong, Chikashi Takeda, Atsuko Shiraki, Shino Matsukawa, Satoshi Shimizu, Shinichi Kai

Department of Anesthesia, Kyoto University Hospital, Kyoto, Japan

\section{*Corresponding author:}

Toshiyuki Mizota

Department of Anesthesia, Kyoto University Hospital, 54 Shogoin-Kawahara-Cho, Sakyo$\mathrm{Ku}$, Kyoto 606-8507, Japan

Tel: +81-75-751-3433, Fax: +81-75-752-3259

E-mail: mizota@kuhp.kyoto-u.ac.jp

\section{Conflicts of interest: None.}

Funding: This work was supported in part by the JSPS KAKENHI (grant number 16K20092; TM, principle investigator). The funding source played no role in the study design, the collection, analysis and interpretation of data, the writing of the report, and the decision to submit the article for publication.

Abbreviations: AKI, acute kidney injury; CKD, chronic kidney disease; eGFR, estimated glomerular filtration rate; SCr, serum creatinine; CI, confidence interval. 


\begin{abstract}
Purpose: We conducted a retrospective cohort study to determine incidences of transient and persistent acute kidney injury (AKI) after major abdominal surgery and their impacts on long-term outcome.
\end{abstract}

Materials and Methods: We enrolled 3,751 patients undergoing major abdominal surgery. Postoperative AKI was classified as transient or persistent based on the return of serum creatinine to the non-AKI range within 7 days post-surgery. Primary outcome was mortality within 1 year. We used multivariable Cox proportional hazard regression analysis to assess independent associations between AKI type and mortality.

Results: Most patients with AKI were classified as transient (84\%). Compared to patients without AKI, both patients with transient and persistent AKI demonstrated elevated 1-year mortality rates [adjusted hazard ratio (95\% confidence interval): 2.01 (1.34-2.93); $\mathrm{P}=0.001$, and 6.20 (3.00-11.43); $\mathrm{P}<0.001$, respectively] and greater risk of chronic kidney disease progression at 1 year [adjusted odds ratio (95\% confidence interval): 3.87 (2.12-7.08) and 23.70 (9.64-58.22), respectively; both $\mathrm{P}<0.001]$.

Conclusions: Although most AKI cases after major abdominal surgery recover completely within 7 days, even these patients with transient AKI are at higher risk for 1-year mortality and chronic kidney disease progression compared to patients without AKI.

Key words: acute kidney injury, abdominal surgery, postoperative complication, chronic kidney disease 


\section{Introduction}

Acute kidney injury (AKI) is a leading cause of perioperative morbidity and mortality. It occurs in $6 \%-13 \%$ of patients undergoing non-cardiac surgery [1-3] and is associated with higher mortality [4,5]. Moreover, AKI is associated with an increased risk of chronic kidney disease (CKD) development or CKD progression in various clinical settings [6-11]. In our clinical experience, postoperative AKI is often transient. It has been suggested that transient AKI represents a temporary reduction in glomerular filtration rate without structural kidney injury, whereas persistent AKI reflects structural renal tubular damage [12]. However, recent studies in hospitalized patients have found that even transient AKI is associated with long-term morbidity and mortality [13-15].

To date, few studies have evaluated the incidence of transient AKI after non-cardiac surgery [16], and the prognostic value of transient AKI in non-cardiac surgery patients is not well validated. Moreover, the impact of transient AKI on long-term kidney function is not known. Revealing the incidence of transient AKI and its impact on outcome in surgical populations may help identify patients with poor postoperative course and provide appropriate treatment alternatives.

Therefore, we conducted a retrospective cohort study to determine the incidence and impact on long-term outcomes of transient and persistent AKI after major abdominal surgery. We hypothesized that the majority of AKI after major abdominal surgery would be transient. In addition, we investigated the associations between AKI type and subsequent long-term outcomes including progression of CKD and mortality. 


\section{Materials and Methods}

\subsection{Study design, setting, and patients}

We conducted a single-center retrospective cohort study at Kyoto University Hospital, an 1,121-bed teaching hospital in Japan. The study protocol was approved by the Ethics Committee of Kyoto University Hospital (approval number: R0672, July $26^{\text {th }}$, 2016), and the requirement for informed consent was waived. This article adheres to the Strengthening the Reporting of Observational Studies in Epidemiology guidelines [17]. We recruited patients using the database created for our previous study investigating the relationship between intraoperative oliguria and AKI after major abdominal surgery [18]. Patients 18 years or older who underwent major abdominal surgery (liver, colorectal, gastric, pancreatic, or esophageal resection) under general anesthesia at Kyoto University Hospital from March 2008 to April 2015 were eligible for inclusion. Exclusion criteria included concurrent cardiac or urological procedures and patients with end-stage renal disease [i.e., estimated glomerular filtration rate $(\mathrm{eGFR})<15 \mathrm{~mL} / \mathrm{min} / 1.73 \mathrm{~m}^{2}$ as determined using a formula validated in Japan [19] or a receipt of hemodialysis]. We also excluded patients who died within 7 days of surgery because mortality in these cases appeared unrelated to AKI, and assessing the incidences of transient and persistent AKI or their impacts on subsequent outcomes are not relevant in this population.

\subsection{Data collection}

The research database for our previous study [18] included age, sex, preoperative comorbidities, American Society of Anesthesiologists Physical Status, preoperative serum creatinine ( $\mathrm{SCr}$ ) (the most recent SCr level measured before surgery), type of surgery, $\mathrm{SCr}$ during the 7 days after surgery, postoperative length of hospital stay, and in-hospital mortality. In addition, data on survival time during the year after surgery and SCr at 3 months 
and 1 year post-surgery were collected from the electronic medical record system.

\subsection{Outcomes and exposure}

The primary outcome was mortality during 1-year follow-up. Secondary outcomes included in-hospital mortality, hospital length of stay, and CKD progression at 3 months and 1 year after surgery. CKD progression was defined as worsening of eGFR category (stage $1, \geq 90$ $\mathrm{ml} / \mathrm{min} / 1.73 \mathrm{~m}^{2}$; stage $2,60-89 \mathrm{ml} / \mathrm{min} / 1.73 \mathrm{~m}^{2}$; stage $3 \mathrm{a}, 45-59 \mathrm{ml} / \mathrm{min} / 1.73 \mathrm{~m}^{2}$; stage $3 \mathrm{~b}$, $30-44 \mathrm{ml} / \mathrm{min} / 1.73 \mathrm{~m}^{2} ;$ stage $4,15-29 \mathrm{ml} / \mathrm{min} / 1.73 \mathrm{~m}^{2}$; and stage $5,<15 \mathrm{ml} / \mathrm{min} / 1.73 \mathrm{~m}^{2}$ ) coupled with a $\geq 25 \%$ reduction in eGFR from baseline according to the Kidney Disease: Improving Global Outcomes 2012 Clinical Practice Guideline for the Evaluation and Management of Chronic Kidney Disease [20]. We calculated eGFR at 3 months and 1 year using the lowest SCr measured at postoperative days 8-90 and postoperative days 276-365, respectively. If SCr was not measured during these periods, the first value measured after the indicated period was used.

The exposure of interest was AKI type (transient or persistent). AKI was defined as any stage of AKI according to the Kidney Disease: Improving Global Outcomes guidelines [21]. The AKI stages were based on the SCr concentration measured during the 7 days after surgery. Preoperative SCr was considered as the baseline. Transient AKI was defined as SCr values returning to below the AKI range within the first 7 days after surgery and persistent AKI as incomplete reduction of SCr at 7 days after surgery. This assessment window of 7 days was chosen because SCr levels fall below the AKI range within 7 days in the majority of patients with AKI and it may allow for comparison with previous studies [16,22].

\subsection{Statistical analyses}

Continuous variables are presented as median [interquartile range] and compared using the Mann-Whitney U test. Categorical variables are presented as numbers (percentage) and 
compared using the Pearson chi-square test or Fisher exact test as appropriate. Competing risk analysis was used to compare length of hospital stay between groups. Median length of hospital stay and 95\% confidence intervals (CIs) were obtained using the cumulative incidence estimates of discharge while alive accounting for death as a competing risk, and Gray's test was used to assess differences between groups. Survival was plotted on KaplanMeier curves and compared between groups by the log-rank test. Moreover, Cox proportional hazard regression analysis was performed to investigate the independent relationship between AKI type and risk of death during 1-year follow-up. The following variables were included in the multivariable model based on clinical relevance: age, sex, preoperative comorbidities (hypertension, diabetes mellitus, CKD, and active congestive heart failure), type of surgery, emergency status, duration of surgery, intraoperative blood loss, and the need for intraoperative vasopressor infusion. CKD was defined as a preoperative eGFR level of $<60$ $\mathrm{ml} / \mathrm{min} / 1.73 \mathrm{~m}^{2}$. Multivariable logistic regression analysis was performed to assess the independent relationship between AKI type and CKD progression among patients with 1-year survival. On the basis of clinical relevance and a literature search for factors associated with CKD and its progression [23], seven variables [age, sex, body mass index, preoperative comorbidities (hypertension, diabetes mellitus, and active congestive heart failure), and preoperative eGFR] were included in the multivariable model. Moreover, the adjusted hazard ratio for mortality and adjusted odds ratio for CKD progression at 1 year was assessed after stratifying based on the maximal AKI stage.

All eligible patients in the study database were included to maximize statistical power. Our study database included approximately 3,800 eligible surgeries, and we assumed 1-year mortality after abdominal surgery to be $5 \%-6 \%$ on the basis of a published report [24]. We estimated that approximately 20 variables could be included in the Cox proportional hazard regression analysis using our dataset. We planned to conduct a complete patient analysis 
including only patients with complete data on all variables required for multivariable analyses. Such an analysis is feasible only if $<5 \%$ of patients have missing data [25]. Our data set fulfilled this criterion.

All statistical tests were two tailed, and a P value of $<0.05$ was considered statistically significant. All statistical analyses were performed using the statistical program $\mathrm{R}$ (http://cran.r-project.org). 


\section{Results}

\subsection{Baseline characteristics}

Figure 1 shows the flow diagram of this study. A total of 3,804 index major abdominal surgeries were identified in the study database. After excluding 39 patients with end-stage renal disease preoperatively and 6 patients who died within 7 days postoperatively, 3,759 patients were eligible for the study. Among these patients, $8(0.2 \%)$ had missing data on variables required for multivariable analyses. Patients with missing values accounted for less than $5 \%$, so we conducted complete patient analysis including 3,751 patients.

The median age of the study participants was 66 years [interquartile range: 56-73] and 2,323 patients $(61.9 \%)$ were male. AKI occurred in 258 patients $(6.9 \%)$ of which $216(5.8 \%)$ were classified as transient and $42(1.1 \%)$ as persistent. Table 1 shows the preoperative patient characteristics and operative variables stratified by AKI type. Patients who developed AKI had a higher AKI risk index, more blood loss, and were more likely to receive intraoperative red blood cell transfusion and intraoperative vasopressor infusion. There were no statistically significant differences in clinical variables between patients with transient and persistent AKI except that patients with persistent AKI were younger than those with transient AKI. Table 2 shows maximal AKI stage stratified by AKI type. The maximal AKI stage was lower in patients with transient AKI (of which $89.4 \%$ were in stage 1).

\subsection{Clinical events according to AKI type}

Clinical events during the 1-year follow-up are listed in Table 3. Patients with AKI (both transient and persistent) showed higher in-hospital mortality, longer length of hospital stay, higher mortality at 3 months and 1 year post-surgery, and more frequent CKD progression at 3 months and 1 year post-surgery than patients without AKI. Kaplan-Meier curve analysis revealed significantly higher death rates in both transient and persistent AKI groups 
compared to patients without AKI $(\mathrm{P}<0.001$ for both comparisons; Figure 2). Survival probabilities at 1 year were $94.6 \%(93.8 \%-95.4 \%)$ for patients without AKI, 83.7\% $(77.8 \%-$ $88.1 \%)$ for those with transient AKI, and 74.5\% (57.6\%-85.5\%) for those with persistent AKI. Multivariable Cox proportional hazard regression analysis revealed that both transient and persistent AKI independently associated with higher mortality rate during 1-year followup even after adjustment for confounders [adjusted hazard ratio $(95 \% \mathrm{CI})$ for transient $\mathrm{AKI}$ : 2.01 (1.34-2.93), $\mathrm{P}=0.001$; adjusted hazard ratio $(95 \% \mathrm{CI})$ for persistent AKI: 6.20 (3.0011.43), $\mathrm{P}<0.001$ ] (Table 4). The association between transient/persistent AKI and mortality was found to be qualitatively preserved when stratified by the maximal AKI stage (Supplementary Table 1).

\subsection{Associations between AKI type and CKD progression at 1 year}

Among the 1-year survivors, 3.2\% exhibited CKD progression at 1 year (Table 3). Most (77.8\%) of the patients with CKD progression demonstrated reduced eGFR level by one eGFR category. Four patients developed CKD stage 5 at 1 year; all these patients had CKD stage 4 preoperatively (Supplementary Table 2). Multivariable logistic regression analysis revealed that both transient and persistent AKI were independently associated with CKD progression at 1 year after adjustment for known risk factors [adjusted odds ratio $(95 \% \mathrm{CI})$ for transient AKI: 3.87 (2.12-7.08), P < 0.001; adjusted odds ratio $(95 \% \mathrm{CI})$ for persistent AKI: 23.70 (9.64-58.22), $\mathrm{P}<0.001]$ (Table 5). The association between transient/persistent $\mathrm{AKI}$ and CKD progression at 1 year was qualitatively preserved when stratified by the maximal AKI stage (Supplementary Table 1). 


\section{Discussion}

Of the 258 patients exhibiting AKI after major abdominal surgery, $84 \%$ were classified as transient according to the return of SCr value to the non-AKI range within the first 7 days post-surgery. However, like persistent AKI patients, these transient AKI patients demonstrated higher in-hospital mortality, longer hospital stay, and higher mortality rate during 1-year follow-up than non-AKI patients. Moreover, both transient and persistent AKI were associated with higher risk of CKD progression at 1 year independent of known risk factors for $\mathrm{CKD}$ and its progression. These data suggest that even temporary AKI after major abdominal surgery can increase the risk of mortality and CKD progression. Therefore, patients with AKI require careful follow-up even if $\mathrm{SCr}$ values return to the non-AKI range shortly after surgery.

Of those patients exhibiting postoperative AKI following major abdominal surgery, the vast majority (84\%) recovered completely within 7 days of surgery. This result is consistent with a previous study in which $90 \%$ of patients with AKI after gastric resection exhibited full renal recovery within 7 days [16]. In contrast, full renal recovery within 7 days has been reported in only $43 \%-65 \%$ of hospitalized patients who develop AKI [13-15]. This discrepancy may be explained by differences in the pathophysiology of AKI between hospitalized patients and postoperative patients. For instance, the most common causes of AKI in hospitalized patients include sepsis and heart failure [26, 27]. In contrast, the intraoperative period is unique in that both anesthesia and surgery combine to affect renal function [28].

To the best of our knowledge, this is the first study demonstrating that postoperative transient $\mathrm{AKI}$ is associated with $\mathrm{CKD}$ progression in patients undergoing non-cardiac surgery. A recent meta-analysis reported the association between the duration of AKI and outcomes including long-term mortality and incident CKD [29]. However, only two studies included in this systematic review reported on the risk for CKD in association with the duration of AKI; of 
these, one study reported that even transient $\mathrm{AKI}$ is associated with $\mathrm{CKD}$ development in hospitalized patients [30], whereas the other study targeted patients undergoing cardiac surgery but was underpowered to assess the impact of transient AKI on CKD development [31]. Kim et al. [16] reported that postoperative transient AKI after gastric surgery was associated with higher long-term mortality; however, postoperative transient AKI was not associated with CKD development in their study, in contrast to the results of our study. There are several possible explanations for this apparent inconsistency. First, our study included patients with preoperative CKD, whereas the study by Kim et al. included only patients with normal preoperative renal function. The impact of transient AKI on CKD progression may be less severe in patients with normal preoperative renal function compared to those with preoperative renal dysfunction. Second, we used CKD progression to assess deterioration of renal function, whereas the study by Kim et al. used new CKD development. These difference in outcome definitions may contribute to the discrepancy in results. Early detection of CKD progression is extremely important because CKD-associated complications and progression to kidney failure can be delayed or even prevented through early introduction of appropriate interventions, including blood pressure and glycemic control [20]. Our data that postoperative transient AKI is associated with CKD progression suggest that even patients with transient $\mathrm{AKI}$ are at higher risk of CKD progression, and therefore require careful follow-up and may benefit from interventions to prevent $\mathrm{CKD}$ progression even after complete recovery of renal function. One possible explanation for this association with CKD progression is that many patients with transient AKI have structural kidney injury despite complete recovery of SCr. Alternatively, AKI may enhance the vulnerability to subsequent CKD due to other factors, such as hypertension and hyperglycemia. As expected, persistent AKI was more strongly associated with outcomes including CKD progression and long-term survival than transient AKI. These data suggest that early 
identification of AKI and intensive treatment aimed at early recovery of renal function may benefit patients with postoperative AKI, although verification by future studies is required. Limitations of our study are as follows. This was a single-center study, which may reduce the generalizability of our results. Our study included patients undergoing major abdominal surgery, so it is unclear whether our findings can be extrapolated to patients undergoing other surgeries. Considering the study design, effects of unmeasured confounding variables on the observed associations cannot be ruled out, despite of our best efforts to adjust for confounding factors. For example, data on albuminuria/proteinuria, the use of nephrotoxins, the use of angiotensin-converting enzyme inhibitors or angiotensin II receptor blockers, and the etiology of AKI for the enrolled patients were not available. Serum creatinine was not measured every day during the 7 days after surgery in all patients, so the incidence of transient AKI may have been underestimated. Nevertheless, the incidence of transient AKI in our study was as high as $84 \%$. Finally, although an assessment window of 7 days was selected to distinguish between transient and persistent AKI, previous studies have applied different thresholds for determining the duration of AKI. Recently, the Acute Disease Quality Initiative proposed a definition of persistent AKI as the continuance of AKI beyond $48 \mathrm{~h}$ [32]. The optimal duration of AKI to define transient and persistent AKI remains to be elucidated. 


\section{Conclusions}

Although most patients with postoperative AKI after major abdominal surgery completely recover within the first 7 days, even patients with transient AKI are at higher risk for longterm mortality and CKD progression compared to those without AKI. Patients with AKI require careful follow-up even if SCr values return quickly to the non-AKI range after surgery. 


\section{Acknowledgments}

We are grateful to Daisuke Sutou, Tomoko Hosoya, Yasushi Matsumoto, and Masaru

Watanabe (Medical Information Systems Section, Management Division, Kyoto University

Hospital, Kyoto, Japan) for their assistance in data collection. 


\section{References}

[1] Abelha FJ, Botelho M, Fernandes V, Barros H. Determinants of postoperative acute kidney injury. Crit Care 2009;13:R79.

[2] Biteker M, Dayan A, Tekkeşin Aİ, Can MM, Taycı İ, İlhan E, et al. Incidence, risk factors, and outcomes of perioperative acute kidney injury in noncardiac and nonvascular surgery. Am J Surg 2014;207:53-9.

[3] O'Connor ME, Kirwan CJ, Pearse RM, Prowle JR. Incidence and associations of acute kidney injury after major abdominal surgery. Intensive Care Med 2016;42:521-30.

[4] Bihorac A, Yavas S, Subbiah S, Hobson CE, Schold JD, Gabrielli A, et al. Long-term risk of mortality and acute kidney injury during hospitalization after major surgery. Ann Surg $2009 ; 249: 851-8$.

[5] Bell S, Dekker FW, Vadiveloo T, Marwick C, Deshmukh H, Donnan PT, et al. Risk of postoperative acute kidney injury in patients undergoing orthopaedic surgery--development and validation of a risk score and effect of acute kidney injury on survival: observational cohort study. BMJ 2015;351:h5639.

[6] Newsome BB, Warnock DG, McClellan WM, Herzog CA, Kiefe CI, Eggers PW, et al. Long-term risk of mortality and end-stage renal disease among the elderly after small increases in serum creatinine level during hospitalization for acute myocardial infarction. Arch Intern Med 2008;168:609-16.

[7] Wald R, Quinn RR, Luo J, Li P, Scales DC, Mamdani MM, et al. Chronic dialysis and death among survivors of acute kidney injury requiring dialysis. JAMA 2009;302:1179-85. [8] Ishani A, Xue JL, Himmelfarb J, Eggers PW, Kimmel PL, Molitoris BA, et al. Acute kidney injury increases risk of ESRD among elderly. J Am Soc Nephrol 2009;20:223-8. 
[9] James MT, Hemmelgarn BR, Wiebe N, Pannu N, Manns BJ, Klarenbach SW, et al.

Glomerular filtration rate, proteinuria, and the incidence and consequences of acute kidney injury:a cohort study. Lancet 2010;376:2096-103.

[10] James MT, Ghali WA, Knudtson ML, Ravani P, Tonelli M, Faris P, et al. Associations between acute kidney injury and cardiovascular and renal outcomes after coronary angiography. Circulation 2011;123:409-16.

[11] Ishani A, Nelson D, Clothier B, Schult T, Nugent S, Greer N, et al. The magnitude of acute serum creatinine increase after cardiac surgery and the risk of chronic kidney disease, progression of kidney disease, and death. Arch Intern Med 2011;171:226-33.

[12] Lameire N, Van Biesen W, Vanholder R. Acute renal failure. Lancet 2005;365:417-30. [13] Uchino S, Bellomo R, Bagshaw SM, Goldsmith D. Transient azotaemia is associated with a high risk of death in hospitalized patients. Nephrol Dial Transplant 2010;25:1833-9. [14] Wi J, Ko YG, Kim JS, Kim BK, Choi D, Ha JW, et al. Impact of contrast-induced acute kidney injury with transient or persistent renal dysfunction on long-term outcomes of patients with acute myocardial infarction undergoing percutaneous coronary intervention. Heart 2011;97:1753-7.

[15] Choi JS, Kim YA, Kim MJ, Kang YU, Kim CS, Bae EH, et al. Relation between transient or persistent acute kidney injury and long-term mortality in patients with myocardial infarction. Am J Cardiol 2013;112:41-5.

[16] Kim CS, Bae EH, Ma SK, Kweon SS, Kim SW. Impact of transient and persistent acute kidney injury on chronic kidney disease progression and mortality after gastric surgery for gastric cancer. PLoS One 2016;11:e0168119.

[17] Vandenbroucke JP, von Elm E, Altman DG, Gøtzsche PC, Mulrow CD, Pocock SJ, et al. Strengthening the Reporting of Observational Studies in Epidemiology (STROBE): explanation and elaboration. Epidemiology. 2007;18:805-35. 
[18] Mizota T, Yamamoto Y, Hamada M, Matsukawa S, Shimizu S, Kai S. Intraoperative oliguria predicts acute kidney injury after major abdominal surgery. $\mathrm{Br} \mathrm{J}$ Anaesth 2017;119:1127-34.

[19] Matsuo S, Imai E, Horio M, Yasuda Y, Tomita K, Nitta K, et al. Revised equations for estimated GFR from serum creatinine in Japan. Am J Kidney Dis 2009;53:982-92.

[20] Kidney Disease: Improving Global Outcomes Chronic Kidney Disease Work Group. KDIGO 2012 clinical practice guideline for the evaluation and management of chronic kidney disease. Kidney Int Suppl 2013;3:1-150.

[21] Kidney Disease: Improving Global Outcomes Acute Kidney Injury Work Group. KDIGO clinical practice guideline for acute kidney injury. Kidney Int Suppl. 2012;2:1-138. [22] Fidalgo P, Ahmed M, Meyer SR, Lien D, Weinkauf J, Kapasi A, et al. Association between transient acute kidney injury and morbidity and mortality after lung transplantation: a retrospective cohort study. J Crit Care 2014;29:1028-34.

[23] Echouffo-Tcheugui JB, Kengne AP. Risk models to predict chronic kidney disease and its progression: a systematic review. PLoS Med 2012;9: e1001344.

[24] Brynjarsdottir ED, Sigurdsson MI, Sigmundsdottir E, Möller PH, Sigurdsson GH. Prospective study on long-term outcome after abdominal surgery. Acta Anaesthesiol Scand 2018;62:147-58.

[25] Royston P, Moons KG, Altman DG, Vergouwe Y. Prognosis and prognostic research: Developing a prognostic model. BMJ 2009;338: b604.

[26] Bellomo R, Kellum JA, Ronco C. Acute kidney injury. Lancet 2012;380:756-66.

[27] Prowle JR, Bellomo R. Sepsis-associated acute kidney injury: macrohemodynamic and microhemodynamic alterations in the renal circulation. Semin Nephrol 2015;35:64-74. [28] Goren O, Matot I. Perioperative acute kidney injury. Br J Anaesth 2015;115(Suppl 2): ii3-14. 
[29] Mehta S, Chauhan K, Patel A, Patel S, Pinotti R, Nadkarni GN, et al. The prognostic importance of duration of AKI: a systematic review and meta-analysis. BMC Nephrol 2018;19:91.

[30] Heung M, Steffick DE, Zivin K, Gillespie BW, Banerjee T, Hsu CY, et al. Acute Kidney Injury Recovery Pattern and Subsequent Risk of CKD: An Analysis of Veterans Health Administration Data. Am J Kidney Dis 2016;67:742-52.

[31] Palomba H, Castro I, Yu L, Burdmann EA. The duration of acute kidney injury after cardiac surgery increases the risk of long-term chronic kidney disease. J Nephrol 2017;30:56772.

[32] Chawla LS, Bellomo R, Bihorac A, Goldstein SL, Siew ED, Bagshaw SM, et al. Acute kidney disease and renal recovery: consensus report of the Acute Disease Quality Initiative (ADQI) 16 Workgroup. Nat Rev Nephrol 2017;13:241-57. 
Table 1. Patient characteristics and operative variables

\begin{tabular}{|c|c|c|c|c|c|}
\hline & $\begin{array}{c}\text { All patients }(\mathrm{n}= \\
3,751)\end{array}$ & No AKI $(n=3,493)$ & $\begin{array}{l}\text { Transient AKI } \\
\qquad(\mathrm{n}=216)\end{array}$ & $\begin{array}{l}\text { Persistent AKI } \\
(\mathrm{n}=42)\end{array}$ & P-value \\
\hline Male sex & $2,323(61.9 \%)$ & $2,112(60.5 \%)$ & $176(81.5 \%)^{*}$ & $35(83.3 \%)^{*}$ & $<0.001$ \\
\hline Diabetes mellitus & $617(16.4 \%)$ & $547(15.7 \%)$ & $54(25.0 \%)^{*}$ & $16(38.1 \%)^{*}$ & $<0.001$ \\
\hline Active congestive heart failure & $63(1.7 \%)$ & $53(1.5 \%)$ & $9(4.2 \%)^{*}$ & $1(2.4 \%)$ & 0.012 \\
\hline Ascites & $311(8.3 \%)$ & $282(8.1 \%)$ & $21(9.7 \%)$ & $8(19.1 \%)^{*}$ & 0.028 \\
\hline AKI risk index & & & $*$ & $*$ & $<0.001$ \\
\hline Class 1 & $1,319(35.2 \%)$ & $1,286(36.8 \%)$ & $29(13.4 \%)$ & $4(9.5 \%)$ & \\
\hline Class 2 & $1,229(32.8 \%)$ & $1,158(33.2 \%)$ & $62(28.7 \%)$ & $9(21.4 \%)$ & \\
\hline Class 3 & $804(21.4 \%)$ & $713(20.4 \%)$ & $75(34.7 \%)$ & $16(38.1 \%)$ & \\
\hline
\end{tabular}


Class 4

Class 5

Type of surgery

Liver

Colorectal

Gastric

Pancreatic

Oesophageal

Complex

Laparoscopic surgery

Emergency surgery

Epidural anaesthesia

Duration of surgery (min)

Intraoperative blood loss (mL)

Net fluid balance during the surgery $(\mathrm{mL})$

Intraoperative red blood cell transfusion

\begin{abstract}
$309(8.2 \%)$
\end{abstract}
$90(2.4 \%)$

$1,189(31.7 \%)$

$1,105(29.5 \%)$

$665(17.7 \%)$

$554(14.8 \%)$

$207(5.5 \%)$

$31(0.8 \%)$

$1,965(52.4 \%)$

$44(1.2 \%)$

1,798 (47.9\%)

353 [259-473]

150 [30-515]

2543 [1831-3487]

$328(8.7 \%)$

\section{$263(7.5 \%)$}

$73(2.1 \%)$

$1,079(30.9 \%)$

$1,053(30.2 \%)$

$627(18.0 \%)$

$510(14.6 \%)$

$197(5.6 \%)$

$27(0.8 \%)$

$1,888(54.1 \%)$

$39(1.1 \%)$

$1,657(47.4 \%)$

347 [256-465]

132 [28-460]

2505 [1815-3449]

$256(7.3 \%)$
$34(15.7 \%)$

$12(28.6 \%)$

$16(7.4 \%)$

$1(2.4 \%)$

$93(43.1 \%)$

$17(40.5 \%)$

$42(19.4 \%)$

$10(23.8 \%)$

$27(12.5 \%)$

$11(26.2 \%)$

$41(19.0 \%)$

$3(7.1 \%)$

$9(4.2 \%)$

$1(2.4 \%)$

$4(1.9 \%)$

$0(0.0 \%)$

$59(27.3 \%)^{*}$

$18(42.9 \%)$

$<0.001$

$5(2.3 \%)$

$0(0.0 \%)$

0.221

\section{$122(56.5 \%)^{*}$}

$19(45.2 \%)$

0.034

444 [340-595]*

446 [315-544]*

$<0.001$

783 [216-1575]*

621 [86-1602]*

$<0.001$

3131 [2259-4319]*

3274 [2402-4053]*

$<0.001$

$63(29.2 \%)^{*}$

$9(21.4 \%)^{*}$

$<0.001$ 
Intraoperative vasopressor infusion

$343(9.1 \%)$

$289(8.3 \%)$

$44(20.4 \%)^{*}$

$10(23.8 \%)^{*}$

$<0.001$

Data are presented as median [interquartile range] or numbers (percentages). ${ }^{*}, \mathrm{P}<0.017$ (significantly different with Bonferroni correction), compared with patients without AKI; $\uparrow, \mathrm{P}<0.017$ (significantly different with Bonferroni correction), compared with patients with transient AKI; AKI, acute kidney injury; ASA-PS, the American Society of Anaesthesiologists physical status; SCr, serum creatinine; eGFR, estimated glomerular filtration rate. 
Table 2. Maximal AKI stage stratified by AKI type

\begin{tabular}{lcc}
\hline Maximal AKI stage & $\begin{array}{c}\text { Transient AKI } \\
(\mathrm{n}=216)\end{array}$ & $\begin{array}{c}\text { Persistent AKI } \\
(\mathrm{n}=42)\end{array}$ \\
\hline Stage 1 & $193(89.4 \%)$ & $22(52.4 \%)$ \\
Stage 2 & $18(8.3 \%)$ & $12(28.6 \%)$ \\
Stage 3 & $5(2.3 \%)$ & $8(19.1 \%)$ \\
\hline
\end{tabular}

Data are presented as numbers (percentages). AKI, acute kidney injury 
Table 3. Clinical outcomes according to AKI type

\begin{tabular}{lccccc}
\hline & All patients & No AKI & Transient AKI & Persistent AKI & P-value \\
\hline In-hospital mortality & $42 / 3,750(1.1 \%)$ & $27 / 3,492(0.8 \%)$ & $9 / 216(4.2 \%)^{*}$ & $6 / 42(14.3 \%)^{* \dagger}$ & $<0.001$ \\
Hospital length of stay (days) & $16(16-16)$ & $16(15-16)$ & $27(23-30)^{*}$ & $38(21-74)^{*}$ & $<0.001$ \\
3-month mortality & $41 / 3,751(1.1 \%)$ & $25 / 3,493(0.7 \%)$ & $9 / 216(4.2 \%)^{*}$ & $7 / 42(16.7 \%)^{* \dagger}$ & $<0.001$ \\
CKD progression at 3-month & $13 / 3,665(0.4 \%)$ & $4 / 3,423(0.1 \%)$ & $3 / 207(1.4 \%)^{*}$ & $6 / 35(17.1 \%)^{* \dagger}$ & $<0.001$ \\
1-year mortality & $217 / 3,751(5.8 \%)$ & $174 / 3,493(5.0 \%)$ & $33 / 216(15.3 \%)^{*}$ & $10 / 42(23.8 \%)^{*}$ & $<0.001$ \\
CKD progression at 1-year & $99 / 3,087(3.2 \%)$ & $74 / 2,902(2.6 \%)$ & $15 / 160(9.4 \%)^{*}$ & $10 / 25(40.0 \%)^{* \dagger}$ & $<0.001$
\end{tabular}

One patient still hospitalized at the time of data collection was excluded from the analyses of in-hospital mortality and hospital length of stay.

Forty-one patients who died within 3 months after surgery and 45 patients with missing SCr data at 3 months after surgery were excluded from the analysis of CKD progression at 3 months. Additionally, 217 patients who died within 1 year after surgery and 447 patients with missing SCr data at 1 year after surgery were excluded from the analysis of CKD progression at 1 year. The median time [interquartile range] from the surgery to the evaluation of CKD progression at 3-month and 1-year was 17 [10-40] and 335 [298-366] days, respectively. Denominators indicate the total number of patients. Hospital length of stay was presented as median $(95 \%$ confidence interval). *, $\mathrm{P}<0.017$ (significantly different with Bonferroni correction), compared to patients without AKI. †, P $<0.017$ (significantly different with Bonferroni correction), compared to patients with transient $\mathrm{AKI}$. AKI, acute kidney injury; CKD, chronic kidney disease. 
Table 4. Multivariable Cox proportional hazard regression analysis assessing independent association between AKI type and mortality

Adjusted hazard ratio $(95 \% \mathrm{CI}) \quad$ P-value

\begin{tabular}{|c|c|c|}
\hline \multicolumn{3}{|l|}{ AKI type } \\
\hline No AKI & 1 (Reference) & \\
\hline Transient AKI & $2.01(1.34-2.93)$ & 0.001 \\
\hline Persistent AKI & $6.20(3.00-11.43)$ & $<0.001$ \\
\hline Age (per 10 years) & $1.37(1.20-1.58)$ & $<0.001$ \\
\hline Male sex & $1.11(0.82-1.52)$ & 0.502 \\
\hline Hypertension & $0.96(0.71-1.29)$ & 0.803 \\
\hline Diabetes mellitus & $1.26(0.91-1.73)$ & 0.154 \\
\hline Chronic kidney disease & $1.11(0.78-1.54)$ & 0.557 \\
\hline Active congestive heart failure & $1.84(0.76-3.73)$ & 0.160 \\
\hline \multicolumn{3}{|l|}{ Type of surgery } \\
\hline Colorectal & 1 (Reference) & \\
\hline Liver & $1.64(1.02-2.64)$ & 0.039 \\
\hline Gastric & $1.60(0.96-2.67)$ & 0.070 \\
\hline Pancreatic & $2.72(1.70-4.34)$ & $<0.001$ \\
\hline Esophageal & $3.70(2.08-6.56)$ & $<0.001$ \\
\hline Complex & $8.42(3.91-18.12)$ & $<0.001$ \\
\hline Emergency surgery & $0.70(0.04-3.24)$ & 0.714 \\
\hline Duration of surgery (per $1 \mathrm{~h}$ ) & $1.11(1.06-1.17)$ & $<0.001$ \\
\hline Intraoperative blood loss (per $100 \mathrm{~mL}$ ) & $1.01(1.01-1.02)$ & $<0.001$ \\
\hline The need for intraoperative vasopressor & $1.14(0.77-1.65)$ & 0.512 \\
\hline infusion & & \\
\hline
\end{tabular}


AKI, acute kidney injury. 
Table 5. Multivariable logistic regression analysis assessing independent associations between AKI type and CKD progression at 1 year

$$
\text { Adjusted odds ratio }(95 \% \mathrm{CI}) \quad \text { P-value }
$$

\begin{tabular}{lcc}
\hline AKI type & 1 (Reference) & \\
No AKI & $3.87(2.12-7.08)$ & $<0.001$ \\
$\quad$ Transient AKI & $23.70(9.64-58.22)$ & $<0.001$ \\
$\quad$ Persistent AKI & $1.39(1.14-1.70)$ & $<0.001$ \\
Age (per 10 years) & $0.76(0.49-1.18)$ & 0.223 \\
Male sex & $0.96(0.90-1.03)$ & 0.239 \\
Body mass index & $1.62(1.02-2.56)$ & 0.039 \\
Hypertension & $1.54(0.94-2.54)$ & 0.086 \\
Diabetes mellitus & $0.73(0.16-3.30)$ & 0.681 \\
Active congestive heart failure & $1.20(1.10-1.32)$ & $<0.001$ \\
Preoperative eGFR (per $\left.10 \mathrm{~mL} / \mathrm{min} / 1.73 \mathrm{~m}^{2}\right)$ & \\
\hline AKI, acute kidney injury; CKD, chronic kidney disease; eGFR, estimated glomerular filtration \\
rate
\end{tabular}


Figure captions

Figure 1. Flow diagram of the study participants. AKI, acute kidney injury.

\section{Identified in the database $(n=6,899)$}

Non-major abdominal surgery $(n=2,823)$

$\longrightarrow$ Including a cardiac or urologic procedure $(n=25)$

Not an index case $(n=247)$

\section{Medical records reviewed $(n=3,804)$}

End-stage renal disease $(n=39)$

Died within 7 days $(n=6)$

Missing data $(n=8)$

Included in the analysis $(n=3,751)$

$$
\begin{gathered}
\text { No AKI } \\
(n=3,493)
\end{gathered}
$$

Transient AKI $(n=216)$

$$
(n=42)
$$


Figure 2. Kaplan-Meier curve of patients without AKI, with transient AKI, or with persistent AKI. No AKI vs. Transient AKI: $\mathrm{P}<0.001$, No AKI vs. Persistent AKI: $\mathrm{P}<0.001$, Transient AKI vs. Persistent AKI: $\mathrm{P}=0.066$. AKI, acute kidney injury.

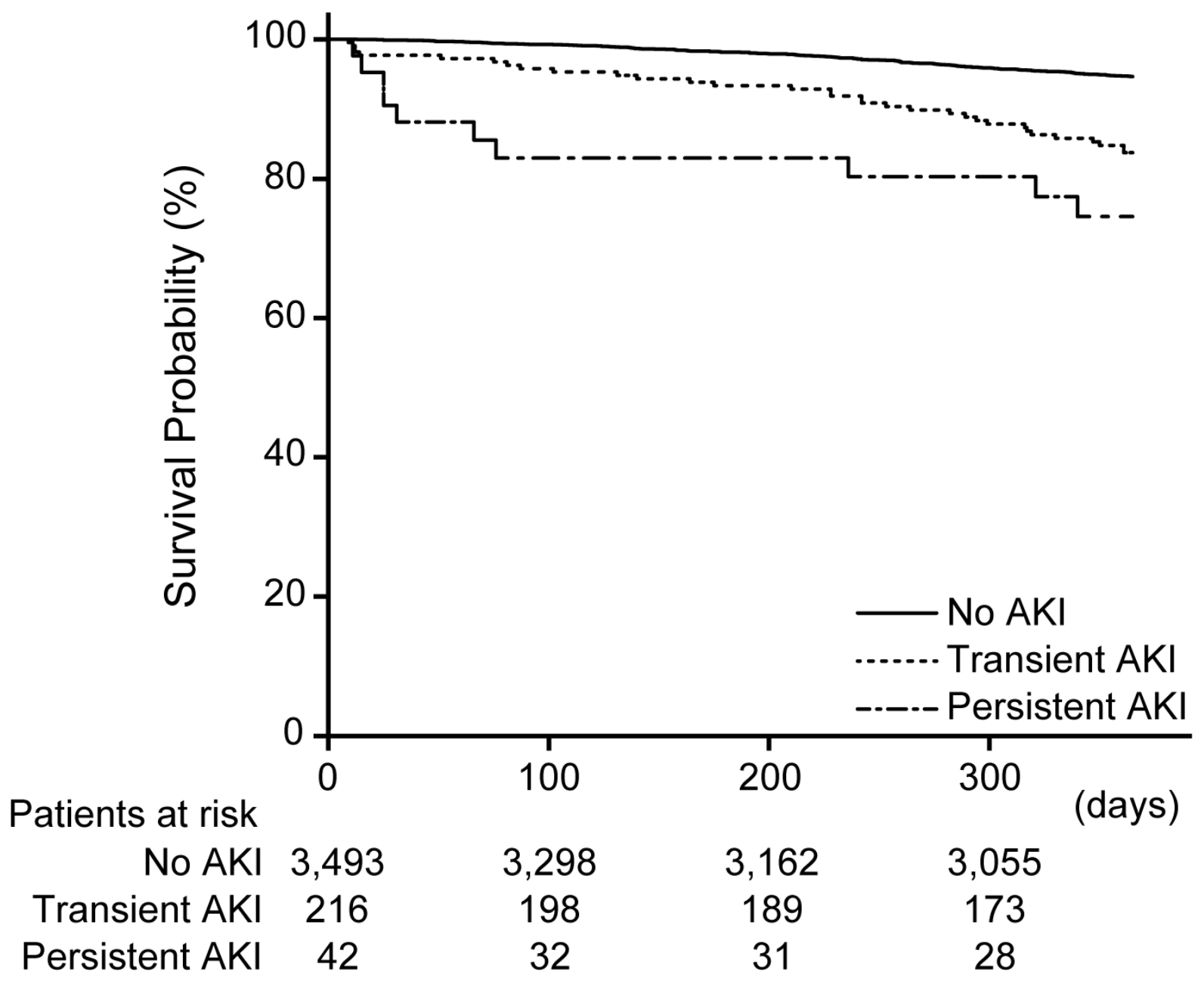


Supplementary Table 1. Association of AKI type and outcomes stratified by the maximal AKI stage

\begin{tabular}{|c|c|c|c|c|}
\hline & \multicolumn{2}{|c|}{ Transient AKI } & \multicolumn{2}{|c|}{ Persistent AKI } \\
\hline & Adjusted association & P-value & Adjusted association & P-value \\
\hline \multicolumn{5}{|l|}{ Mortality } \\
\hline Stage 1 AKI & $1.97(1.32-2.96)$ & $<0.001$ & $1.13(0.16-8.16)$ & 0.903 \\
\hline Stage 2-3 AKI & $2.19(0.69-6.96)$ & 0.183 & $11.38(5.61-23.08)$ & $<0.001$ \\
\hline \multicolumn{5}{|c|}{ CKD progression at 1 year } \\
\hline Stage $1 \mathrm{AKI}$ & $3.59(1.86-6.93)$ & $<0.001$ & $13.40(4.21-42.70)$ & $<0.001$ \\
\hline Stage 2-3 AKI & $5.37(1.44-20.00)$ & 0.012 & $63.1(13.6-293.0)$ & $<0.001$ \\
\hline
\end{tabular}

Mortality was reported as hazard ratios and CKD progression at 1 year as odds ratios; AKI, acute kidney injury; CKD, chronic kidney disease 
Supplementary Table 2. Preoperative eGFR category and eGFR category at 1 year of patients demonstrating CKD progression at 1 year

\begin{tabular}{|c|c|c|c|c|c|c|c|c|c|c|c|c|c|c|c|}
\hline \multirow[b]{2}{*}{ Preoperative eGFR category } & \multicolumn{5}{|c|}{$\begin{array}{l}\text { No AKI } \\
(\mathrm{n}=74)\end{array}$} & \multicolumn{5}{|c|}{$\begin{array}{l}\text { Transient AKI } \\
\qquad(\mathrm{n}=15)\end{array}$} & \multicolumn{5}{|c|}{$\begin{array}{l}\text { Persistent AKI } \\
(\mathrm{n}=10)\end{array}$} \\
\hline & 1 & 2 & $3 a$ & $3 b$ & 4 & 1 & 2 & $3 a$ & $3 b$ & 4 & 1 & 2 & $3 a$ & $3 b$ & 4 \\
\hline \multicolumn{16}{|l|}{ eGFR category at 1 year } \\
\hline 2 & 26 & - & - & - & - & 1 & - & - & - & - & 2 & - & - & - & - \\
\hline $3 a$ & 1 & 27 & - & - & - & 0 & 3 & - & - & - & 1 & 2 & - & - & - \\
\hline $3 b$ & 1 & 9 & 5 & - & - & 0 & 4 & 4 & - & - & 0 & 3 & 0 & - & - \\
\hline 4 & 0 & 1 & 0 & 2 & - & 1 & 0 & 0 & 1 & - & 1 & 0 & 0 & 0 & - \\
\hline 5 & 0 & 0 & 0 & 0 & 2 & 0 & 0 & 0 & 0 & 1 & 0 & 0 & 0 & 0 & 1 \\
\hline
\end{tabular}

Most patients with CKD progression demonstrated worsening of eGFR by one eGFR category. The proportions of patients with no AKI,

transient AKI and persistent AKI who demonstrated reduced eGFR level by $\geq 2$ eGFR categories were $16.2 \%, 33.3 \%$, and $50.0 \%$, respectively.

Four patients developed CKD stage 5 at 1 year. eGFR, estimated; AKI, acute kidney injury. 